\title{
Right Atrial Mass Mimicking a Myxoma as a First Presentation of Antiphospholipid Syndrome
}

\author{
Nael Al-Sarraf, FRCSC, Abouelmakarem Abdelmoaty, MD, Sameh Abu alam, MD, \\ Jamal Al-Fadhli, FRCSC
}

Department of Cardiac Surgery, Chest Diseases Hospital, Kuwait

\section{ABSTRACT}

Antiphospholipid syndrome is characterized by recurrent spontaneous abortion, recurrent arterial/venous thrombosis, and thrombocytopenia. The presentation of an atrial mass mimicking a myxoma in this syndrome is exceedingly rare, leading to misdiagnosing of the condition and further risking recurrence due to lack of anticoagulation. In this report, we present such a case that was mistakenly thought to be a myxoma.

\section{INTRODUCTION}

Antiphospholipid syndrome is an autoimmune disorder characterized by recurrent spontaneous abortion, recurrent arterial and venous thrombosis, and thrombocytopenia. The presence of cardiac manifestation is rare. In this case, we share a rare presentation of antiphospholipid syndrome, which presented as an atrial mass mimicking a myxoma.

\section{CASE REPORT}

A 26-year-old female presented to her local hospital with dyspnea and palpitations. She previously was healthy and previously underwent Cesarean section, just 15 months prior. She was a non-smoker and not taking any medications. She had two previous pregnancies with no abortions. Her heart rate was 100 beats per minute (sinus tachycardia), and she was saturating $100 \%$ on room air. Physical examination was unremarkable. Her blood workup revealed a healthy liver and renal profile. However, she had thrombocytopenia and elevated activated partial thromboplastin time (APTT). Her D-Dimers were raised on presentation. The chest radiograph was normal. Computerized tomography (CT) of the chest showed segmental right lung pulmonary embolism (PE). Because of her palpitations, transthoracic echo was done which showed a right atrial (RA) mass close to the inferior vena cava (IVC) and prolapsing in and out through the tricuspid valve with dilated right atrium and severe tricuspid regurgitation (TR). She urgently was transferred to

Received fune 1, 2019; received in revised form August 24, 2019; accepted August 29, 2019.

Correspondence: Dr Nael Al-Sarraf, Al-fabriah, PO Box 1134, Postal Code 46312, Kuwait; +965-98882921 (e-mail: trinityq8@hotmail.com). our center for cardiac surgery intervention for the presumed atrial myxoma. She was commenced on low molecular weight heparin for her PE. Repeat echo confirmed the right atrial mass with $2.5 \mathrm{~cm}^{*} 2.1 \mathrm{~cm}$ close to the RA/IVC junction with severe TR and normal pulmonary artery pressure (Figure 1A to Figure 1C). No other masses were seen. The patient was reviewed by a hematologist, who thought the thrombocytopenia was possibly secondary to the myxoma and no specific therapy was advised. Due to her embolization to the lung, we proceeded urgently for surgery. Sternotomy and excision of right atrial mass, closure of patent foramen ovale, and tricuspid valve replacement (size 27 Magna Ease Bioprosthetic valve) were performed. The
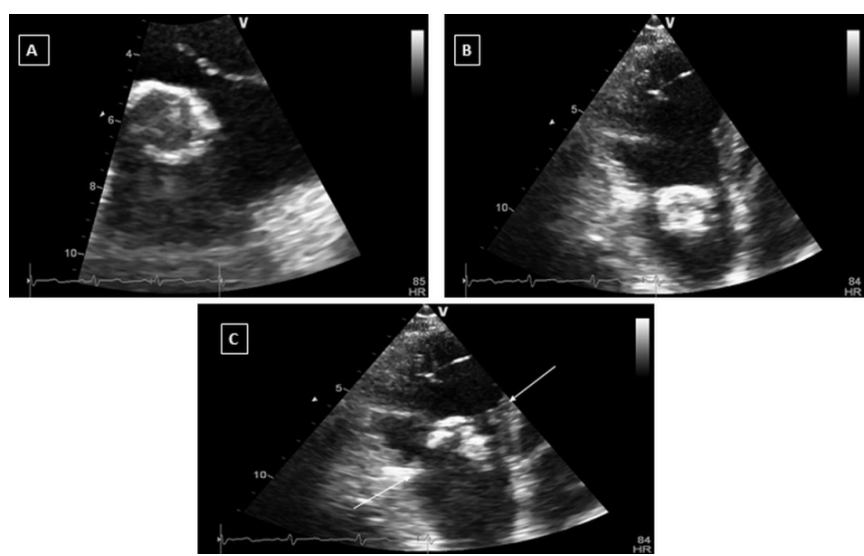

Figure 1. Echocardiography appearance of the right atrial mass. A, and $B$, parasternal short axis view of the mass. C: Relation of the mass to tricuspid valve annulus (arrow).

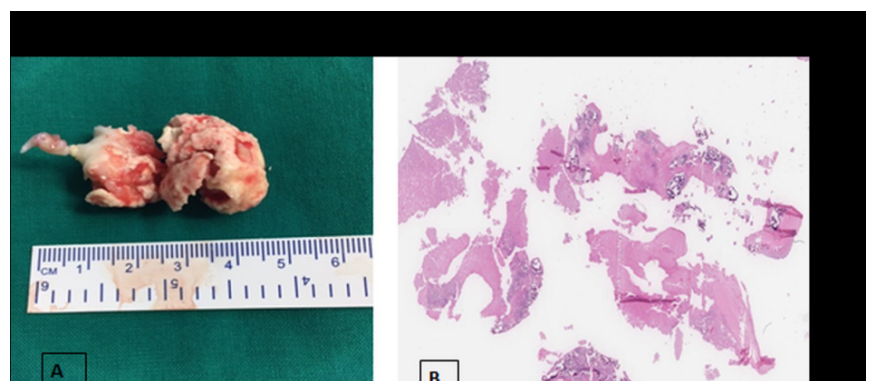

Figure 2. A, Intraoperative appearance of the right atrial mass following resection. B, histological appearance of calcified hematoma (Hematoxylin and Eosin stain *100 magnification power). 
A brief summary of some reported cases of antiphospholipid syndrome with atrial mass

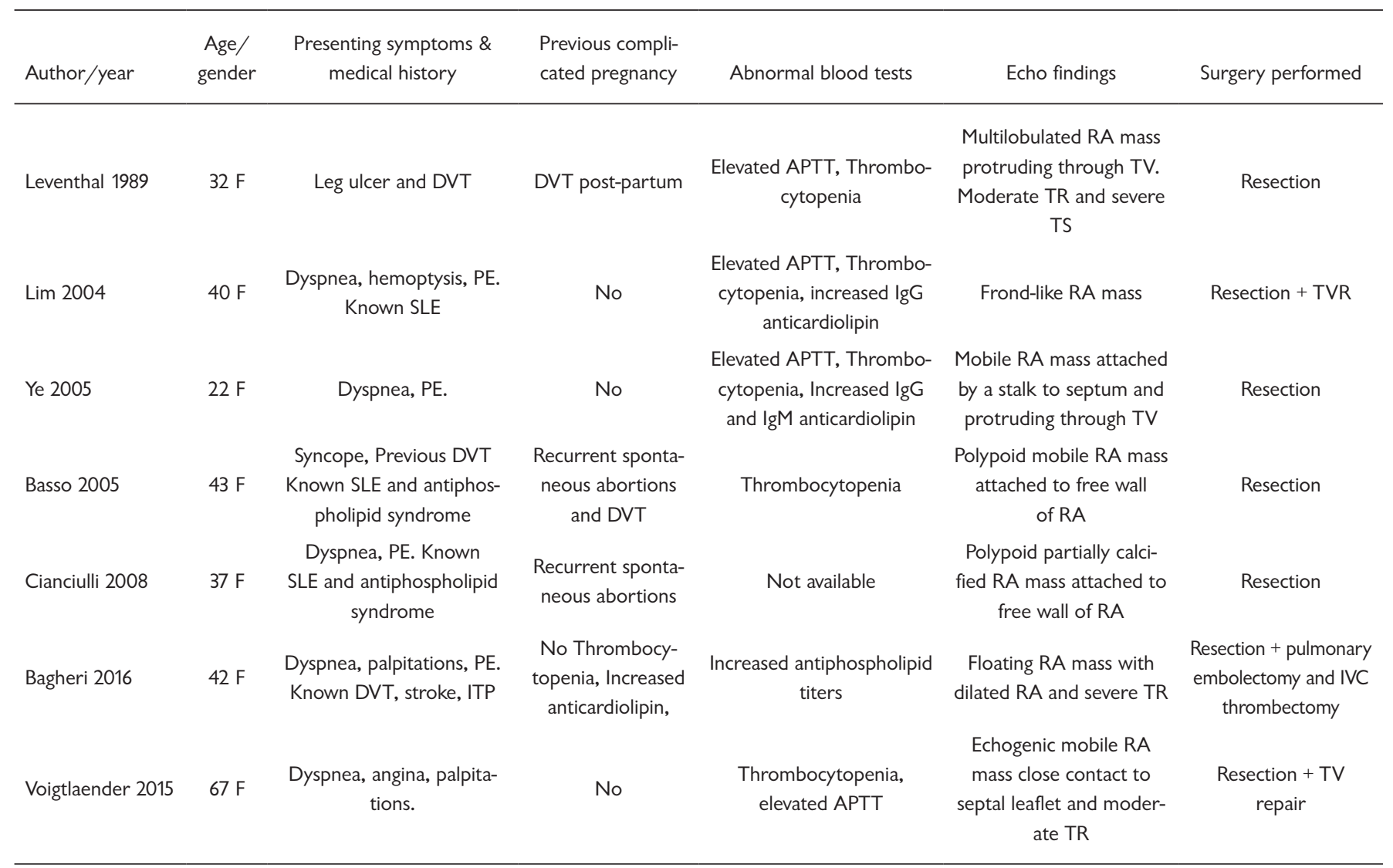

APTT: activated partial thromboplastin time. ITP: Idiopathic Thrombocytopenic purpura. PE: Pulmonary embolism. DVT: Deep Venous Thrombosis. SLE: Systemic Lupus erythematosus. IVC: Inferior vena cava. TVR: Tricuspid Valve Replacement. TV: tricuspid valve. TR: tricuspid regurgitation. RA: right atrium.

tricuspid valve was found to be severely destroyed by the mass with severe TR. The mass was excised with its stalk (Figure 2A). It was found to be near the septal leaflet, and the valve leaflets were fibrotic. There was calcification in the mass. Postoperatively, the patient did well and was discharged home uneventfully 7 days later. She was commenced on oral anticoagulation with coumadin for her PE for 6 months with a target international normalized ratio (INR) between 2-3 and low dose Aspirin (for the bioprosthetic valve). Her platelet count recovered to normal levels by postoperative Day 4 . Her pathology showed calcified right atrial hematoma with no tumor seen (Figure 2B). After 6 months of treatment with oral anticoagulation, a repeat CT study was performed and showed complete resolution of PE. Repeat echocardiography showed a well-functioning tricuspid valve with no recurrence of the mass. At that point, her oral anticoagulant was stopped, and she was referred to a hematologist to rule out a hypercoagulable state. Her hematological workup showed a healthy level of Antithrombin III, free protein $S$, and protein $C$ with normal activated protein $C$ resistance (APCR). However, lupus anticoagulant was strongly positive and a repeated sample 1 month later also was strongly positive. Anticardiolipin IgG and $\operatorname{IgM}$ were both positive. In addition, Anti-beta 2- glycoprotein-1 IgG and IgM also were strongly positive, suggesting antiphospholipid syndrome. The patient had no other features of antiphospholipid syndrome. She was then recommenced on oral anticoagulation with coumadin to prevent any future thromboembolic events. Her clinical situation has improved, and she has remained asymptomatic for 8 months.

\section{DISCUSSION}

Antiphospholipid syndrome is an autoimmune disorder characterized by recurrent arterial and venous thrombosis, recurrent spontaneous abortion, and thrombocytopenia [Ye 2005; Basso 2005]. The serological diagnosis is based on elevated titer of lupus anticoagulant or anticardiolipin antibodies, which are a family of autoantibodies predominantly directed against negatively charged phospholipids. Antiphospholipid syndrome can be seen alone (referred to as primary) or secondary, when it occurs in association with other diseases, such as systemic lupus erythematosus [Cianciulli 2008]. Thrombocytopenia is a common finding with a reported prevalence of $20 \%$ to $40 \%$ [Voigtlaender 2015]. The most common clinical features of antiphospholipid syndrome are 
deep venous thrombosis (32\%), PE (9\%), and valvular abnormalities (11\% to $32 \%$ of cases). Other cardiac features include coronary artery disease, dilated cardiomyopathy, and pulmonary hypertension [Bagheri 2016]. However, the occurrence of a right atrial mass at the initial presentation is exceedingly rare [Ye 2005]. Only 10 previous case reports have been published in English literature, with similar presentations to our case. A summary of some of these cases [Ye 2005; Basso 2005; Cianciulli 2008; Voigtlaender 2015; Bagheri 2016; Leventhal 1989; Lim 2004] is shown in Table 1.

The mainstay of treatment is anticoagulation to prevent thromboembolic disease. The role of surgery here often is indicated when the mass is suspected of being a cardiac tumor, such as a myxoma.

\section{CONCLUSION}

In patients presenting with a right atrial mass, thrombocytopenia and prolonged APTT should raise the suspicion of an antiphospholipid syndrome associated with cardiac thrombosis. Long-term anticoagulation is required if appropriate test results confirm this syndrome.

\section{REFERENCES}

Bagheri A, Bagheri J. 2016. Concurrence of pleural effusion and inferior vena cava thrombus in Antiphospholipid syndrome complicated by intracardiac thrombus. Res Cardiovasc Med; 5:e33696.

Basso C, Bottio T, Rubino M, et al. 2005. Antiphospholipid syndrome and right atrial mass. J Thorac Cardiovasc Surg; 130:1462-3.

Cianciulli TF, Saccheri MC, Redruello HJ, et al. 2008. Right atrial thrombus mimicking myxoma with pulmonary embolism in a patient with systemic lupus erythematosus and secondary antiphospholipid syndrome. Tex Heart Inst J; 35:454-7.

Leventhal LJ, Borofsky MA, Bergey PD, Schumacher HR Jr. 1989. Antiphospholipid antibody syndrome with right atrial thrombosis mimicking an atrial myxoma. Am J Med; 87:111-3.

Lim E, Wicks I, Roberts LJ. 2004. Intracardiac thrombosis complicating antiphospholipid antibody syndrome. Intern Med J; 34:135-7.

Voigtlaender M, Conradi L, Hinsch A, Langer F. 2015. Right Atrial Thrombosis in Antiphospholipid Syndrome with Secondary Immune Thrombocytopenia. Thorac Cardiovasc Surg Rep; 4:40-3.

Ye ZX, Yu WC, Hsueh CM, et al. 2005. Antiphospholipid syndrome presenting as intracardiac thrombus with pulmonary embolism. Circ J; 69:1290-2. 